\title{
PEER: A Progressive Energy Efficient Routing Protocol for Wireless Ad Hoc Networks
}

\author{
Jinhua Zhu and Xin Wang \\ Department of Computer Science and Engineering \\ State University of New York at Buffalo, Buffalo, NY 14260 \\ \{jzhu2, xwang8\}@cse.buffalo.edu
}

\begin{abstract}
Many minimum energy (energy efficient) routing protocols have been proposed so far. However, few effort has been spent on the routing overhead, route setup time, and route maintenance issues associated with such protocols. This paper first shows that the minimum energy routing schemes in the literature could fail without considering the routing overhead involved and the node mobility. It then proposes a more accurate analytical model to track the energy consumption and the impact of packets errors, and a simple energy-efficient routing scheme to improve the performance in mobility scenarios. The simulation results indicate that the PEER-based energy efficient routing has significantly higher performance than that of a normal energybased routing scheme.
\end{abstract}

\section{INTRODUCTION}

In wireless ad hoc networks, mobile devices are often battery powered. But current battery technique still could not support the devices to work long enough. In addition, changing the battery may not be feasible in some application scenarios, such as sensor networks in hostile environment. Therefore, energy saving schemes are very important in wireless ad hoc networks. Since mobile devices are getting smaller and more energy efficient, communication energy cost becomes a much significant part in the total energy consumed. Therefore energy efficient communication scheme is one of the most effective ways to save energy.

In wireless network, the transmitted signal is attenuated at the rate of $1 / d^{n}$, where $d$ is the distance to the sender and $n$ is the path loss exponent between 2 and 6 . Then the basic energy efficient scheme would be to adjust the transmission power according to the distance between the sender and the receiver instead of using the constant maximum transmission power. This is called power control scheme. However, this is not optimal in terms of end-to-end energy consumption. To achieve the optimal solution, many energy efficient routing protocols have been proposed[1]-[8]. These protocols can be generally classified into two categories: Minimum Energy routing protocols[1]-[6] and Maximizing Network Lifetime routing protocols[7][8]. Minimum Energy routing protocols try to find the most energy efficient path to transmit the data packets from the source to the destination, while Maximizing Network Lifetime routing protocols try to balance the remaining batterypower at each node. Since Minimum Energy routing scheme is also an important part in the most recent Maximizing Network Lifetime routing protocols such as Conditional MaxMin Battery Capacity Routing (CMMBCR) protocol[7] and
Conditional Maximum Residual Packet Capacity (CMRPC) routing protocol[8], we will only concentrate on the minimum energy routing protocols in this paper.

Minimum Energy routing protocols can be further divided into three classes based on the types of link costs: Minimum Total Transmission Power (MTTP), Minimum Total TransCeiving Power (MTTCP), and Minimum Total Reliable Transmission Power (MTRTP) protocols. MTTP protocols use the transmission power as the link metric and search for the path with minimum total transmission power between the source and the destination. Authors in [1] modified the Dijkstra's Shortest path algorithm to obtain the minimum total transmission power path. PARO in [5] also used transmission power as the link cost, however it targets at the energy savings between any two neighboring nodes. In this scheme, one or more intermediate nodes elect to forward packets on behalf of the neighboring source-destination pair to reduce the transmission power. MTTCP protocols use the transmission power as well as the receiving power as the link cost. Authors in [3] used distributed Bellman-Ford algorithm to obtain the minimum total transceiving power path. However, the first two classes of protocols did not consider the energy consumption because of data packet retransmission. Authors in [4] proposed MTRTP protocol. This protocol uses the total transmission power for transmitting the data packets from one node to its neighboring node reliably as the link cost. Our work in [6] proposed a new link cost for reliable transmission that includes the energy consumption for data packets as well as that for signaling packets in MAC layer.

Most of previous work concentrated on the link costs. Once a new link cost was derived, then the traditional shortest path routing protocols, such as AODV, DSR, and Bellman-ford, can be modified with the new link cost. However, there are some problems with such straightforward modification. First, the routing overhead for the route discovery is very high, which consumes a lot of energy. Second, the route setup time is very long. Third, the route maintenance scheme is not suitable for dynamic environments, such as mobility scenarios. We will explain these issues in more details in next section.

To address these issues, we propose a Progressive Energy Efficient Routing (PEER) protocol. Contrary to other energyefficient routing protocols that try to find the optimal path at one shot and maintain the route reactively, PEER searches for the more energy efficient path progressively and maintains the route continuously. It first finds a path near the most 
energy efficient path between the source and the destination quickly, and then adjusts the nodes whenever necessary so that the path would be energy efficient all the time. Our performance evaluation shows that PEER achieves less routing overhead, shorter setup time, and great energy efficiency in static scenario as well as the mobile scenario.

The rest of the paper is organized as follows. We explain the observation and motivation of this paper in Section II. In section III, we extend our previous work [6] and propose an efficient way to estimate the accurate link cost. The detailed PEER protocol is descried in Section IV. Performance evaluation is conducted in V. Section VI concludes the work.

\section{OBSERVATION AND MOTIVATION}

Many routing protocols have been proposed for wireless ad hoc networks. These protocols can be generally categorized as: (a) table-driven, (b) on-demand, and (c) hybrid. For tabledriven routing protocols, all nodes need to advertise the routing information periodically so that they can have the up-to-date view of the network. Destination Sequenced Distance Vector (DSDV), Wireless Routing Protocol (WRP), and Cluster Switch Gateway Routing (CSGR) belong to this category. Different from table-driven routing protocols, on-demand routing protocols create the route only when desired by the source node. Some on-demand routing protocols are Ad hoc Ondemand Distance Vector (AODV), Dynamic Source Routing (DSR), and Temporally Ordered Routing Algorithm (TORA). The Zone Routing Protocol (ZRP) is a hybrid protocol with table-driven routing scheme for the intra-zone routing and on-demand routing scheme for the inter-zone routing. Most of energy efficient schemes modified the on-demand routing protocols such as AODV or DSR since there is a lot of routing overhead if using table-driven routing protocols [2]. So, we will only focus on the on-demand energy efficient routing protocols.

For on-demand routing protocols such as AODV, a node will start a route discovery process if it needs a route to a destination. It broadcasts the route request packet and waits for the reply from the destination. The neighboring nodes that receive such route request packet will rebroadcast it, and so on. To reduce the routing overhead, the nodes will only rebroadcast the first route request packet received and discard the following duplicate ones. And the destination node only replies to the first route request packet, too. For example, in Fig 1, both $\mathrm{A}$ and $\mathrm{B}$ are neighboring nodes of $\mathrm{S}$ and $\mathrm{D}$, and $\mathrm{S}$ needs a route to $\mathrm{D}$. So $\mathrm{S}$ broadcasts the route request packet first, and both A and B receive the packet. Assume A broadcasts such packet next, then node S, B and D receive such packet, however node S and B will discard it as they have already received the same route request packet. Therefore the final route is SAD. It is apparent that the routing overhead for these protocols is $\mathrm{O}(n)$, where $n$ is the number of nodes in the network.

Things are quite different for energy efficient routing protocols. The nodes could not simply discard the duplicate route request packets now as they may come from more energy efficient paths. That is, they also need to respond to the route

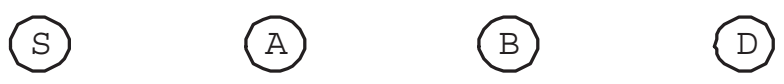

Fig. 1. A linear topology.

request packets from a more energy efficient path. Therefore, the nodes may need to broadcast the same route request packet many times. For the same example in Fig. 1, node B may need to broadcast both the packets from $\mathrm{S}$ and $\mathrm{A}$ if the path SAB is more energy efficient than SB. Based on the BellmanFord algorithm [13], we can obtain that routing overhead for minimum energy efficient routing protocols is $\mathrm{O}\left(n^{2}\right)$ now. Such overhead will consume a lot of energy and network resources, especially when the number of nodes in the network is very large. In addition, the route setup time ${ }^{1}$ is much longer than the on-demand routing protocols. There are two main reasons for this. One is that the energy efficient route has more intermediate nodes than the shortest path in general, so it takes longer time for the route request and route reply packets to go through all the intermediate nodes. The other is that the energy efficient routing protocols have much more routing overhead which can cause more delay at each link. The simulations in GlomoSim verify our observation. From the simulation results in Fig. 5 - 7, it is clear that the routing overhead, energy consumption for routing overhead, and route setup time for the energy efficient routing protocol increase dramatically with the number of nodes in the network, while only linearly for the on-demand routing protocol.

Energy efficient routing protocols tend to use many intermediate nodes so that the distance for each link is very short. This can help reduce the link breakage rate for mobile scenarios since each node has larger moving range without breaking the link. The problem is that the original minimum energy path may no longer be energy efficient before link breakage because of mobility. The worst case would be that the transmission power for each link is at the maximum level when the link distance is at the transmission range. In this case, it would cost much more energy than the on-demand routing protocols since it has much more links in the path. Therefore, the route maintenance scheme in the on-demand routing protocols such as DSR or AODV is not suitable for the energy efficient routing protocols since it maintains the route only when one or more links are broken.

Most existing energy efficient work limited the study to the static scenario. The authors in [2] proposed a scheme for mobility case. In this scheme, a node snoops the data and ACK packet exchanging between the sender and the receiver. If it finds that it is on a lower energy path between the sender and the receiver, it sends out a gratuitous route reply to the source node (DSR) or the sender (AODV) about the lower energy route. We call it "Insert" operation since it would insert a node into the link. However, if every node can move, this scheme will add more and more nodes in the path. The worst case would be that every node in the network is in the path and the transmission power for each link is the maximum power level

\footnotetext{
${ }^{1}$ The time from the source node broadcasts the route request packet until it receives the reply for the desired path such as the minimum energy path.
} 
since there is no more nodes can be added into any link and the link distance is very large. In this case, it is worse than the protocol without using this scheme. PARO [5] also has "Insert" operation. In addition, it can also remove an Inserted node if such node no longer helps to save energy between the link. However, PARO works on a sublayer between the routing and MAC layers. That is, it mainly helps save energy between two neighboring nodes. Even though it can be applied to each link of the path given by the routing protocol, the performance depends on the routing protocol. Even if the routing protocol initially finds a very good path, the nodes can move around so that the path may be very inefficient after some time.

From these observations, we can see that an energy efficient routing protocols should have less overhead, short setup delay, high energy efficiency, and are highly adaptive to mobility. Therefore, we propose our Progressive Energy Efficient Routing (PEER) protocol for wireless ad hoc networks.

\section{ENERGY CONSUMPTION MODEL FOR 802.11}

Link cost is very important in energy efficient routing protocols. Without an accurate link cost, the minimum energy routing protocols could not find the optimal route. In this section, we will first present some physical and MAC layer assumptions used in this paper. Then we extend our model in previous work[6] and propose an efficient way to estimate the link cost.

PEER requires that each node can adjust the transmission power dynamically and retrieve channel information such as noise and received power level. Both are also common assumptions in most energy efficient routing protocols. In addition, it also desires that the MAC protocol can provide reliable hop-by-hop data transmission as retransmission costs a lot of energy. Therefore we use power control 802.11 for MAC protocol, in which RTS and CTS packets are transmitted at the maximum power while DATA and ACK packets are transmitted at the minimum required power level for the receiver to decode correctly. To avoid some collisions, PEER also requires the nodes to set their NAVs (Network Allocation Vector) to the EIF (Extended InterFrame Space) duration if they can sense the signal but can not decode it correctly[10].

We derived an accurate energy consumption model for 802.11 in [6]. Denote the packet sizes of RTS, CTS, DATA, and ACK packets by $N_{r}, N_{c}, N_{d}$ and $N_{a}$, and packet error rates for RTS, CTS, DATA, and ACK packets between node $i$ and $j$ by $p_{r, i, j}, p_{c, j, i}, p_{i, j}$, and $p_{a, j, i}$. In addition, for a variable $x$, denote $1-x$ by $x^{*}$, and the mean value of $x$ by $\bar{x}$. Then the average total transmission power for transmitting a packet from node $i$ to one of its neighboring node, node $j$, is

$$
\overline{P_{T}(i, j)}=\frac{P_{m}\left(\frac{N_{r}}{N_{d}}+\frac{N_{c}}{N_{d}} p_{r, i, j}^{*}\right)}{p_{r, i, j}^{*} p_{c, j, i}^{*} p_{i, j}^{*} p_{a, j, i}^{*}}+\frac{P_{i, j}+P_{j, i} \frac{N_{a}}{N_{d}} p_{i, j}^{*}}{p_{i, j}^{*} p_{a, j, i}^{*}} .
$$

where $P_{m}$ is the maximum power, $P_{i, j}$ and $P_{j, i}$ are the transmission power for DATA and ACK packets respectively. Most of parameters in this model can be easily obtained except the transmission power and the packet error rates.

PEER adopts the transmission power estimation scheme used in [10]. If node $A$ receives a packet transmitted at the maximum power level from node B, such as RTS, CTS and broadcast packets, then node A can calculate the desired transmission power to node $\mathrm{B}, P_{\text {desired }}$, based on the received power, $P_{r}$, and the maximum power level $\left(P_{m}\right)$ as, $P_{\text {desired }}=$ $\frac{P_{m}}{P_{r}} * P r_{\text {thresh }} * c$, where $P r_{\text {thresh }}$ is the minimum necessary received signal strength and $c$ is an constant.

Packet error is mainly caused by collision, interference and noise. Here we distinguish the concept of collision and interference by the carrier sensing zone. If the error is caused by the nodes within the carrier sensing zone, we call it collision, otherwise interference.

It is easy to obtain the interference and noise level since each node can monitor it when the channel is free. With the interference and noise level, we can then calculate the bit error rate based on the received power level and modulation scheme [4]. For example, $B E R=0.5 * \operatorname{erf} c\left(\sqrt{\frac{P_{r}}{N_{o} * f}}\right)$ for BPSK, where $\operatorname{erf} c(x)=1-\int_{0}^{x} e^{-t^{2}} d t, N_{o}$ is the noise spectral density, $P_{r}$ is the received power, and $f$ is transmission bit rate. Once we get the BER, then we can calculate the packet error rate (PER) because of interference and noise (assuming there is no error correction scheme) by $P E R=1-(1-B E R)^{L}$ , where $L$ is the number of bits in the packet.

For 802.11 protocol, most collisions happen during transmitting RTS. Therefore, we only need to consider the packet error rate caused by the collision of RTS packet. Authors in [11] presented a simple way to estimate the collision probability by counting the number of busy/idle slots:

$$
p_{c}(t+1)=\alpha p_{c}(t)+\frac{1-\alpha}{N} \sum_{i=0}^{N-1} C_{t-i}
$$

where $p_{c}(t)$ is the estimated collision probability at time t, $\alpha$ is the remembering rate, and $C_{t-i}$ with $i=0, \ldots, N-1$ are the last $N$ slot samples. $C_{i}$ is equal to 0 if the $i$-th slot is free or the node transmits successful in such slot; otherwise $C_{i}$ is 1 .

Therefore, the packet error rates for CTS, DATA and ACK packets are calculated based on the interference and noise power, receiving power, and the packet size. While for RTS packet, we need to take into account the packet error rate by interference and by collision. Denote the packet error rate for interference and noise by $p_{\text {int }}$, and the packet error rate for collision by $p_{c}$. Then the packet error rate for RTS packet is

$$
p_{r, i, j}=p_{i n t}+p_{c}-p_{i n t} * p_{c}
$$

\section{PEER PROTOCOL}

As a routing protocol, PEER also consists of route discovery process and route maintenance scheme. To solve the problems mentioned in Section II, PEER should search for the energy efficient path efficiently and quickly during route discovery process. In addition, it should maintain the route actively so that it can respond to environment change quickly. In the following, we will show how PEER achieves both goals. 


\section{A. Route Discovery Process}

As shown in Section II, the minimum energy based routing scheme introduces a lot of routing overhead and the setup time is very long. On the other hand, a routing strategy should not get some arbitrary route quickly and rely on a route maintenance scheme to adjust the route later to an energy efficient one as it may cost much more time and overhead to adapt such route and there is no guarantee that such adaption could find a path that could be energy efficient enough as compared to the minimum energy path. Therefore, an optimal strategy is to find an energy efficient path near the most energy efficient path quickly and then a maintenance scheme can adjust the path to be more energy efficient easily. To do this, we first need to know where the most energy efficient path is.

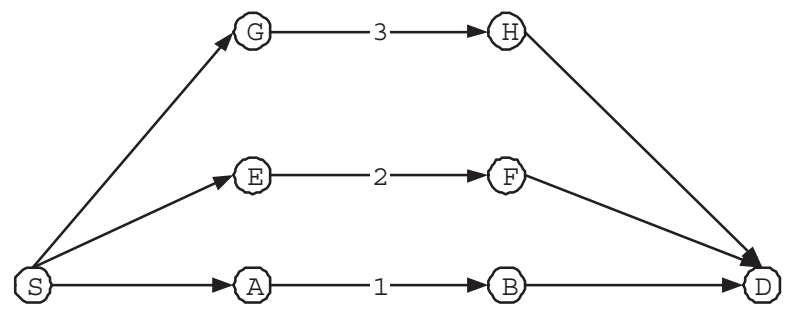

Fig. 2. Three routes between node S and D.

It is easy to prove the following statement given enough nodes $^{2}$ and similar environment (such as same packet error rates for each link) in the network:

The most energy efficient path is on the line between the source and the destination.

Proof: Assume the statement is not true, then at least one node in the most energy efficient path is not on the line between the source and the destination. For such an abnormal node, we can always find the corresponding node on the line between the source and the destination such that the line linking the node pair is vertical to the line between the source and the destination. Therefore, we can have one energy efficient path on the line between the source and destination with the same number of nodes as the most energy efficient path. And such energy efficient path is better than the optimal one as no link is longer than the corresponding one in the most energy efficient path ${ }^{3}$. This contradicts to our assumption. Therefore, the most energy efficient path is on the line between the source and the destination.

Similarly, we can also prove that the $N$-hop most energy efficient path is on the line between the source and the destination given the same conditions, where $N$ is a number smaller than the number of hops in the most energy efficient path. Even though in a practical network the most energy efficient path may not be on the line between the source and the destination, the most energy efficient path is still close to the line. Therefore, to find an energy efficient path near the most energy efficient path would be equivalent to find an

\footnotetext{
${ }^{2} \mathrm{We}$ can assume that there is a node at any place we need in the network.

${ }^{3}$ As we assume the same packet error rates, the energy consumption on each link is determined by the transmission power as well as the link distance.
}

energy efficient path closer to the line between the source and destination.

The quickest way to find a route between two nodes would be through a shortest path routing scheme. However, there is no guarantee that the route obtained by the shortest path routing protocol would be near the line between the source and the destination. From the second statement we proved at the previous paragraph, we can see that the minimum energy path among all the shortest (fewest hops) paths (we call it minimum energy shortest path) would meet our requirement. Denote the set of paths between the source and the destination by $L$, the number of hops for path $l$ by $N_{l}$, and the energy consumption for link $i$ in path $l$ by $E_{l, i}$, then the set of shortest paths $L_{s}$ would be

$$
L_{s}=\arg \min \left(N_{l}\right), l \in L .
$$

And the set of minimum energy shortest paths $L_{m s}$ would be

$$
L_{m s}=\arg \min \left(\sum_{i=1}^{N_{l}} E_{l, i}\right), l \in L_{s} .
$$

Even though there may be more than one minimum energy shortest path in $L_{m s}$, the routing protocol can pick a unique one by some criterion, such as route request packet arriving time.

Based on the previous definition, the basic searching algorithm would be: (1) search for all shortest (fewest hops) paths; (2) pick the minimum energy path(s) among the shortest paths in (1). To implement this algorithm, the route request packet should carry two pieces of information: one is the hop count, the other is the energy consumption. The source node first broadcasts the route request packet with both hop count and energy consumption set to 0 . Once an intermediate node receives a route request packet, it will first update the hop count (increased by 1) and energy consumption (increased by the energy consumption between the sender and itself) information in the route request packet. And then it will rebroadcast such packet only if one of the following conditions holds:

1) The node hasn't received such packet before or the packet comes from a shorter (smaller number of hops) path;

2) The packet comes from a path with the same number of hops as the best path so far, but the energy consumption is less than it.

The first condition make sure the node select the shortest path, while the second condition let it pick the minimum energy path from all shortest paths.

This algorithm also has similar path selection issue as other energy efficient routing protocols. That is, the destination node may receive many route request packets from different possible minimum energy shortest paths, but it could not tell which one is the best until it receives all possible packets. However, the destination node has no knowledge about how many route request packets it will receive. For example, in Fig. 2, assuming all the intermediate nodes (A, B, E, F, G, H) are the neighboring nodes of both $\mathrm{S}$ and $\mathrm{D}$, then there are six shortest (2 hops) paths (SAD, SBD,SED, SFD, SGD, SHD). 
However, the destination node $\mathrm{D}$ has no such information so that it could not pick the minimum energy shortest path even if it already receives all route request packets from all shortest paths. There are several ways to deal with this issue at the destination node. One option is that the destination sends a route reply packet for each route request packet it receives. This method will waste some energy as the destination will send out many route reply messages and the source node might transmit some data packets on less energy efficient path. The other one is that the destination sets up a timer after receiving first route request packet. If it receives another route request packet before timeout, it will reset the timer. Otherwise, it will select the best path so far and reply with a route reply packet when the timer goes off. This method help reduce the energy consumption, but it may increase the route setup time. In this paper, we use the second one.

The minimum energy shortest path may still not be energy efficient enough since it tends to use the long-distance link. Allowing a route to pass through some intermediate nodes may help to save energy. To speed up the route optimization process, this can be done in parallel as the route reply message travels from the destination to the source. When the nodes that are not on the minimum energy shortest path overhear such route reply message, they will check whether they are on a lower energy path between the sender and the receiver (This is similar to the Insert operation we will describe in next subsection). If yes, they will broadcast better route messages and the corresponding nodes will update their routing tables if necessary. For example, in Fig. 2, assuming that the minimum energy shortest path is SAD, then $\mathrm{D}$ will send the route reply message to A, and node B, E, F, G, H overhear it. Assuming that node $\mathrm{B}$ and $\mathrm{F}$ are on the lower energy path between $\mathrm{A}$ and $\mathrm{D}$, then they will broadcast the better route messages. Finally, $A$ and $D$ will include $B$ in the route if the path ABD is more energy efficient than AFD. Then, the route found during the route discovery process is $\mathrm{SABD}$. The better route message overhead can be reduced by using delayed broadcast scheme in [5]. In this scheme, the more energy saving, the quicker a node will broadcast the better route message. Other nodes receiving such message will not broadcast the better route message if they could not save more energy. For the same example, only B will broadcast the better route message.

\section{B. Route Maintenance}

The route obtained in the route discovery process may not be energy efficient enough compared to the minimum energy path. In addition, the network environment can change dramatically, as a result, the previous energy efficient route may no longer be efficient as time goes on. Therefore, the route maintenance phase is very critical for energy efficient routing protocols.

As described in section III, each node can estimate the necessary transmission power and the link cost to one of its neighboring node once it receives RTS, CTS or broadcast packet from such node. PEER requires that each node adds the link cost to the receiver in the IP header as an IP option for each data packet it transmits, and monitors the data

\begin{tabular}{|c|c|c|c|c|c|c|}
\hline (a) & (b) & (c) & (d) & (e) & (f) & (g) \\
\hline A & B & 5 & S1 & D1 & 1 & 0 \\
\hline B & C & 4 & S1 & D1 & 1 & 1 \\
\hline D & B & 3 & S2 & D2 & 3 & 3 \\
\hline F & G & 7 & S3 & D3 & 5 & 4 \\
\hline B & E & 2 & S2 & D2 & 3 & 5 \\
\hline
\end{tabular}

TABLE I

A LINK ENERGY TABLE

packets transmitted in its neighborhood. For each data packet transmitted, received, or overheard by the node, it will record the following information into a link cost table: (a) sender; (b) receiver; (c) link cost between the sender and the receiver; (d) source; (e) destination; (f) IP header ID; (g) the current time. Among these parameters, (a) and (b) can be obtained from the MAC header, while (c) to (f) can be obtained from the IP header. The information for a link will be kept only for a short time for accurate information and reducing storage overhead.

From the link cost table, a node can know how a packet passes through its neighborhood and the total link cost for that. For example, node D's link energy table is in Table I. As the parameters (source, destination, and IP header ID) can identify a packet, we can see in the table that node $\mathrm{D}$ records the path info for three packets: $\mathrm{P} 1(\mathrm{~S} 1, \mathrm{D} 1,1), \mathrm{P} 2(\mathrm{~S} 2, \mathrm{D} 2,3)$ and P3(S3, D3, 5). The first packet (P1) uses two-hop path $(\mathrm{A} \rightarrow \mathrm{B} \rightarrow \mathrm{C})$ in D's neighborhood and the total link cost is $9(5+4)$. The second packet (P2) uses another two-hop path $(\mathrm{D} \rightarrow \mathrm{B} \rightarrow \mathrm{E})$ and the total link cost is $5(3+2)$. The third packet $(\mathrm{P} 3)$ uses one-hop path $(\mathrm{F} \rightarrow \mathrm{G})$ and the link cost is 7 .

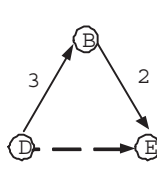

(a)

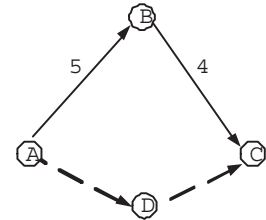

(b)

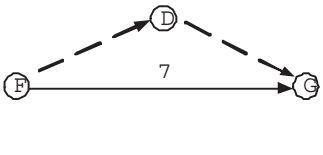

(c)
Fig. 3. Remove, Replace, and Insert.

Based on the information in the link cost table, each node can help improve the local path as well as its corresponding end-to-end path with the three operations (Remove, Replace, and Insert) illustrated for node D in Fig. 3.

(a) Remove

The rule for Remove operation is as follows:

Assume there is a two-hop path $\mathrm{X} \rightarrow \mathrm{A} \rightarrow \mathrm{B}$ with destination $\mathrm{D}$ and total link cost $T$ in X's link cost table. If $X$ finds the link cost between $X$ and $B$ is smaller than that of the two-hop path, it will update its routing table by setting the next hop for destination $D$ to $B$.

In Fig.3(a), node $\mathrm{D}$ has the two-hop path info $(\mathrm{D} \rightarrow \mathrm{B} \rightarrow \mathrm{E})$ from its link energy table with destination D2 and and the total link cost (5) for such path. If node E is one of D's neighboring nodes, D can estimate the link cost to $\mathrm{E}\left(\overline{P_{T}(D, E)}\right)$ from the RTS or CTS packets transmitted by node E. If $\overline{P_{T}(D, E)}<5$, then $\mathrm{D}$ will update its routing table by setting the next hop for 
destination D2 to E. The following packet for destination D2 will go through E directly.

(b) Replace

The rule for Replace operation is as follows:

Assume that there is a two-hop path $\mathrm{A} \rightarrow \mathrm{B} \rightarrow \mathrm{C}$ with destination $\mathrm{D}$ and total link cost $T$ in $\mathrm{X}$ 's link cost table. If $X$ finds the total cost for the path $A \rightarrow X \rightarrow C$ is smaller than that of the two-hop path $A \rightarrow B \rightarrow C, X$ will update its routing table by setting the next hop to destination $D$ to $C$. In addition, it will request $A$ to update A's routing table by setting the next hop to the destination $D$ to itself $(X)$.

In Fig. 3(b), Node $\mathrm{D}$ has the two-hop path info $(\mathrm{A} \rightarrow \mathrm{B} \rightarrow$ C) in its link cost table with the destination D1 and the total link cost (9). If both $\mathrm{A}$ and $\mathrm{C}$ are D's neighboring nodes, D can estimate the link costs to them $\left(\overline{P_{T}(D, A)}, \overline{P_{T}(D, C)}\right)$. If $\overline{P_{T}(D, A)}+\overline{P_{T}(D, C)}<9$, then the path $\mathrm{A} \rightarrow \mathrm{D} \rightarrow \mathrm{C}$ is more energy efficient than $\mathrm{A} \rightarrow \mathrm{B} \rightarrow \mathrm{C}$. So node $\mathrm{D}$ will update its routing table by setting the next hop to destination $\mathrm{D} 1$ to $\mathrm{C}$ and request $A$ to update A's routing table by setting the next hop to destination $\mathrm{D} 1$ to $\mathrm{D}$. If $\mathrm{A}$ accepts the request from $\mathrm{D}$, then the following packets for D1 at node A will be transmitted to node $\mathrm{D}$, and $\mathrm{D}$ will forward them to $\mathrm{C}$. If $\mathrm{A}$ does not accept the request from $\mathrm{D}$, the routing info for destination $\mathrm{D} 1$ at node $\mathrm{D}$ will be purged after some time.

(c) Insert

The rule for Insert operation is as follows:

Assume that there is a one-hop path $\mathrm{A} \rightarrow \mathrm{B}$ with destination $\mathrm{D}$ and total link cost $T$ in $\mathrm{X}$ 's link cost table. If $X$ finds the total cost for the path $A \rightarrow X \rightarrow B$ is smaller than that of one-hop path, it will update its routing table by setting the next hop to destination $D$ to $B$. In addition, $X$ will request A to update A's routing table by setting the next hop to the destination $D$ to itself $(X)$.

In Fig. 3(c), Node $D$ has the one-hop path info $(F \rightarrow G)$ in its link cost table with the destination D3 and the total link cost (7). If both $\mathrm{F}$ and $\mathrm{G}$ are D's neighboring nodes, D can estimate the link costs to them ( $\overline{P_{T}(D, F)}, \overline{P_{T}(D, G)}$ ). If $\overline{P_{T}(D, F)}+\overline{P_{T}(D, G)}<7$, then the path $\mathrm{F} \rightarrow \mathrm{D} \rightarrow \mathrm{G}$ is more energy efficient than $\mathrm{F} \rightarrow \mathrm{G}$. So node $\mathrm{D}$ will update its routing table by setting the next hop to destination D3 to $G$ and request $F$ to update F's routing table by setting the next hop to destination D3 to D.

It is worthwhile to point out that both Replace and Remove operations can work on the path with more than two hops. However, as all nodes on the path in the link cost table should be the neighboring nodes of the monitoring node (such as node $\mathrm{D}$ in previous example) otherwise the monitoring node could not estimate the link costs to them, the probability of such path existing in the link cost table is very low. In addition, the operations on the path with more than two hops sometimes can be replaced by several operations on one-hop or two-hop path. Therefore, we limit both operations on two-hop path only.

Only Replace and Insert operations need the control message. The control messages are only sent out when a better path is noticed so that the maintenance overhead is very low. The control message includes: operation ID, requester ID, destination, next hop, the total link cost for new path. The control message that $\mathrm{D}$ sends to $\mathrm{A}$ for Replace operation is [Replace, D, D1, B, the total link cost for ADC], while the control message that $\mathrm{D}$ sends to $\mathrm{F}$ for Insert operation is [Insert, D, D3, G, the total link cost for FDG]. Once a node receives a control message, it will first check the routing info for the destination in its routing table. If the next hop for such destination is different from that in the control message, it will discard such control message since the route has been changed.

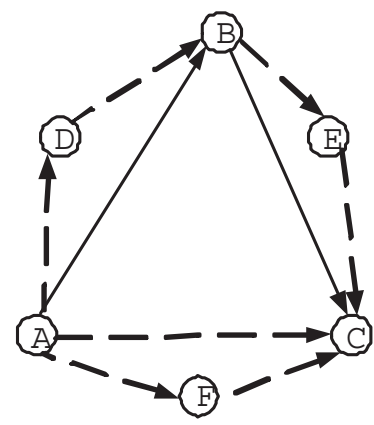

Fig. 4. An undesired improvement

Within these three operations, Insert may have higher priority than the other two since it only needs to check one-hop transmission. This may not be desirable. For example, in Fig 4, node A transmits the data packet to node B. D overhears such data packet so that it sends a packet to A indicating that it can save energy between the link AB. Similarly, node E may be inserted between nodes $\mathrm{B}$ and $\mathrm{C}$. Therefore, the final path will be ADBEC. However, there are two more options, $\mathrm{AC}$ and AFC, and AFC is the best path. So it would be better to let Remove and Replace have higher priority than Insert. In PEER, each node receiving Remove or Insert requests will wait for some time before making the decision. If it has Insert and any other operation request, it will take the other operation. If it has both Remove and Replace operation requests, it will select one by the energy saving percentage. For the same example, node A has the Insert (by node D), Remove, and Replace (by node F) requests, then it will only process Remove and Replace operations. And as AFC is better than $\mathrm{AC}\left(\overline{P_{T}(A, F)}+\overline{P_{T}(F, C)}<\overline{P_{T}(A, C)}\right)$, so it takes the Replace operation.

\section{Performance Evaluation}

We have simulated PEER, MTRTP, as well as normal AODV protocols in Glomosim. We modified AODV with the new link cost derived in [4] for MTRTP protocol. And the power control scheme is also applied to the normal AODV protocol. The network area is $1200(\mathrm{~m}) X 1200(\mathrm{~m})$ and the nodes are randomly distributed over the network. The available transmission power levels are 1,5,10,15, 20, 25, 30, $35 \mathrm{~mW}$. The connection arrival rate follows Poission distribution and the connection duration follows Exponential distribution. The application protocol is CBR (Constant Bit Rate) and the source and destination pairs are randomly selected. The mobility model is random waypoint with 30 -second pause time. Some other default setup parameters are in Table II. 


\begin{tabular}{|c|c|c|c|}
\hline Parameter & Value & Parameter & value \\
\hline Number of Nodes & 60 & Packet Size(byte) & 512 \\
\hline Arrival Rate & 30 & Duration $(\mathrm{min})$ & 6 \\
\hline Max Speed(m/s) & 6 & Min Speed $(\mathrm{m} / \mathrm{s})$ & 0 \\
\hline
\end{tabular}

TABLE II

Default SETUP PARAMETERS

We first studied the route discovery performance for each protocol, and then the energy consumption as well as the retransmission rate in static as well as the mobile scenarios.

\section{A. Routing Overhead and Setup Time}

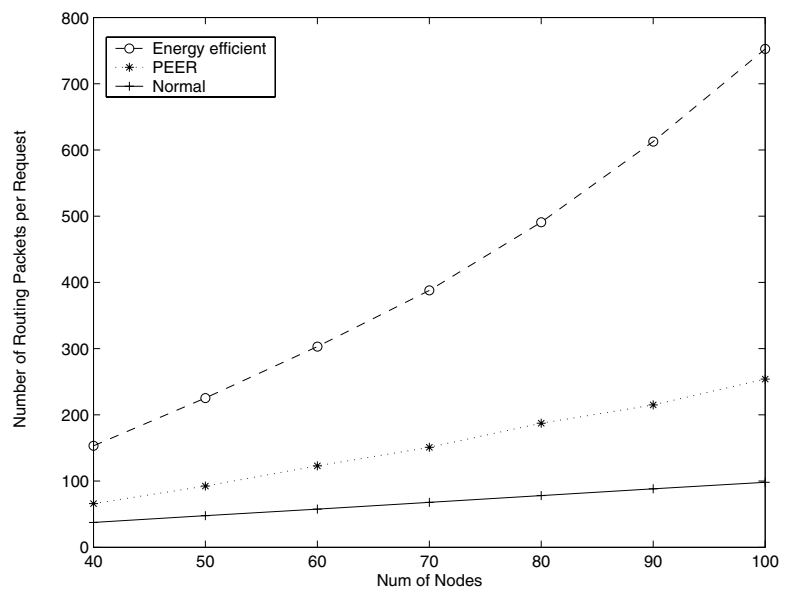

Fig. 5. Routing overhead.

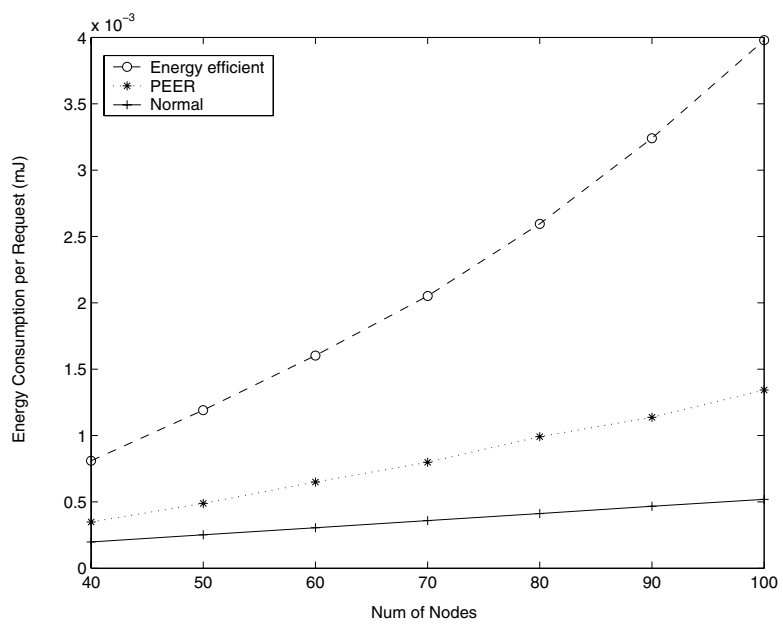

Fig. 6. Energy Consumption for routing overhead.

In this study, we simulated 10,000 connection requests for each protocol and collected the total number of routing packets, total energy consumption, and total setup time on each simulation. The simulation results are in Fig. 5-7.

It is clear from the results that the normal on-demand routing protocol performs the best in terms of routing overhead, energy consumption for routing overhead, and setup time,

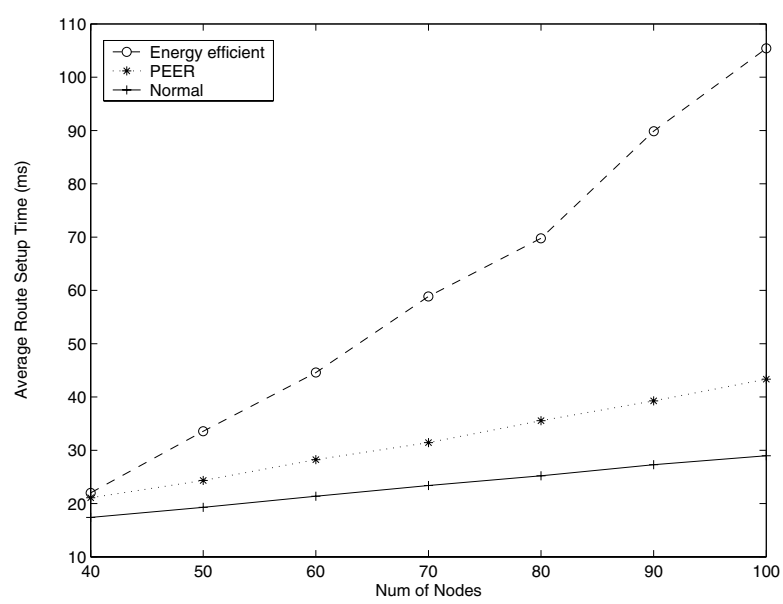

Fig. 7. Route setup time.

followed by PEER and minimum energy routing protocol. Both the routing overhead and setup time for the minimum energy routing protocol are much more than the on-demand routing protocol, and increase dramatically with the number of nodes. That is because the routing overhead for minimum energy routing protocol is $O\left(n^{2}\right)$ ( $n$ is the number of nodes) as discussed in Section II. Therefore the minimum energy routing protocol could not scale well with the number of nodes.

While for PEER protocol, the performance is quite well. Even though both the routing overhead and route setup time are still higher than the on-demand routing protocol, they are much less than the minimum energy routing protocol. Most importantly, both routing overhead and route setup time increase very close to linearly with the number of nodes in the network. So PEER has high scalability with the number of nodes.

\section{B. Static Scenario}

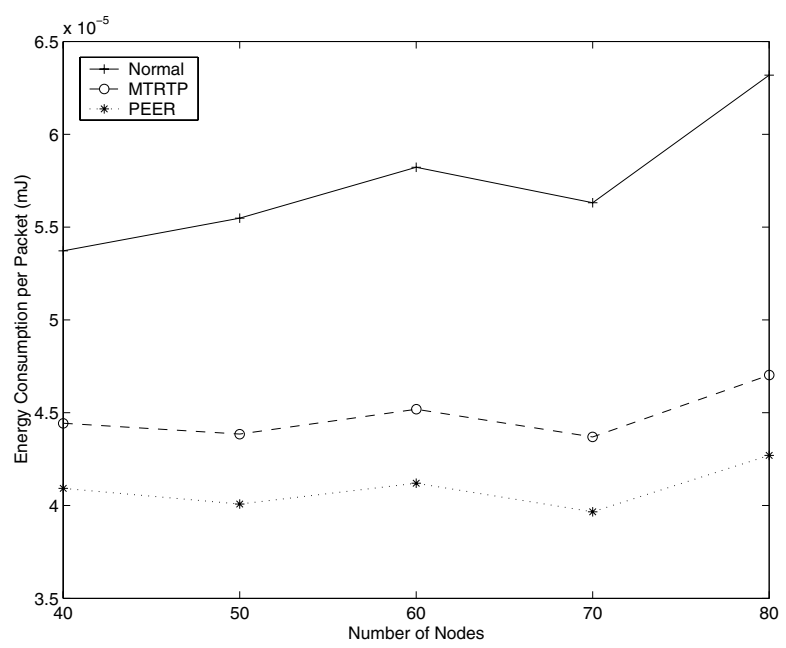

Fig. 8. Different density (static).

In the static scenario, we studied the energy consumption and RTS retransmission rate performance for each protocol 


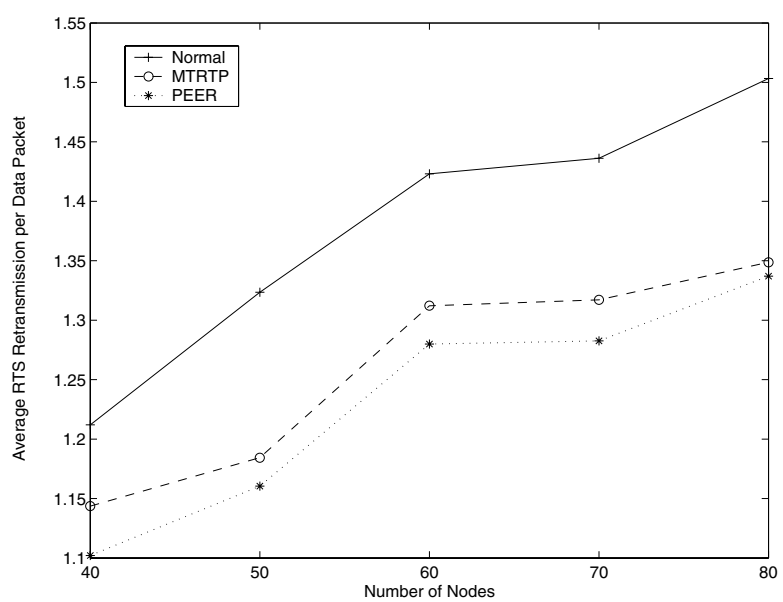

Fig. 9. Different density (static).

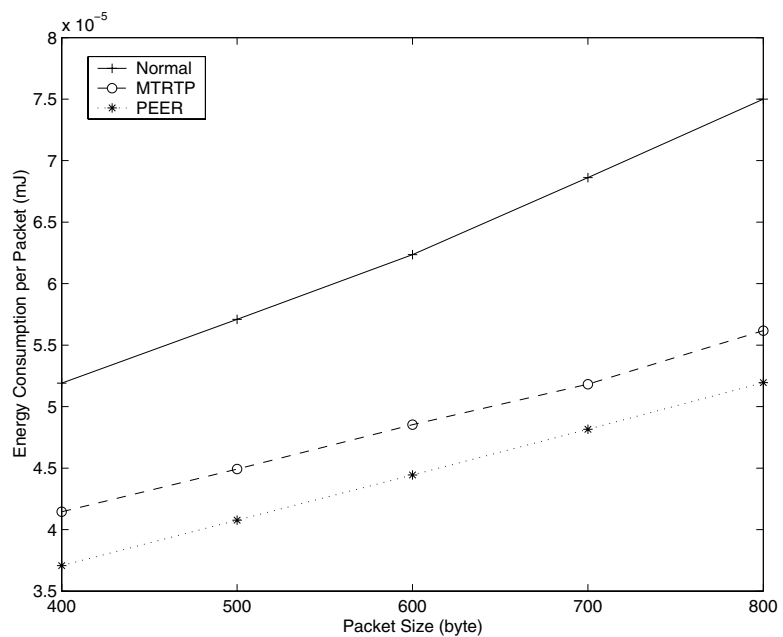

Fig. 10. Different packet size (static).

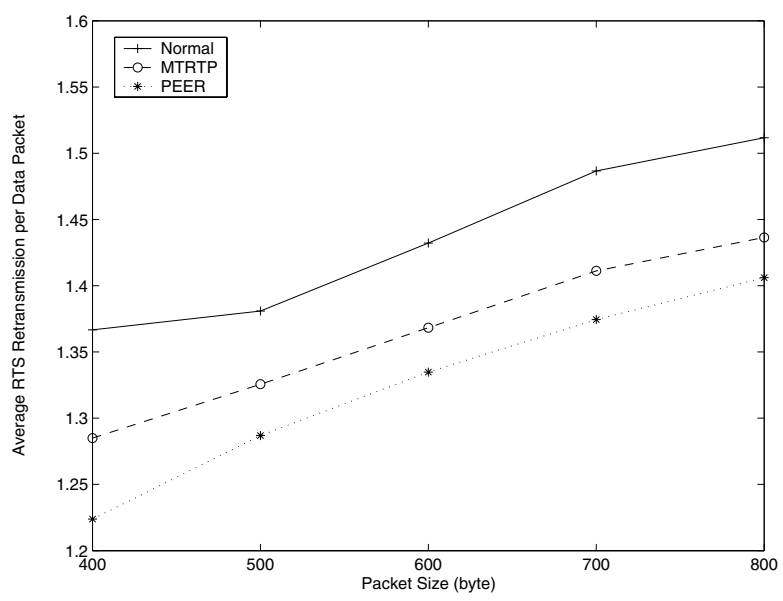

Fig. 11. Different packet size (static).

in three different groups: different density, different packet size, and different connection arrival rate. The simulation time for each protocol is 5 hours. We monitored the total energy consumption, the total number of packets received at all

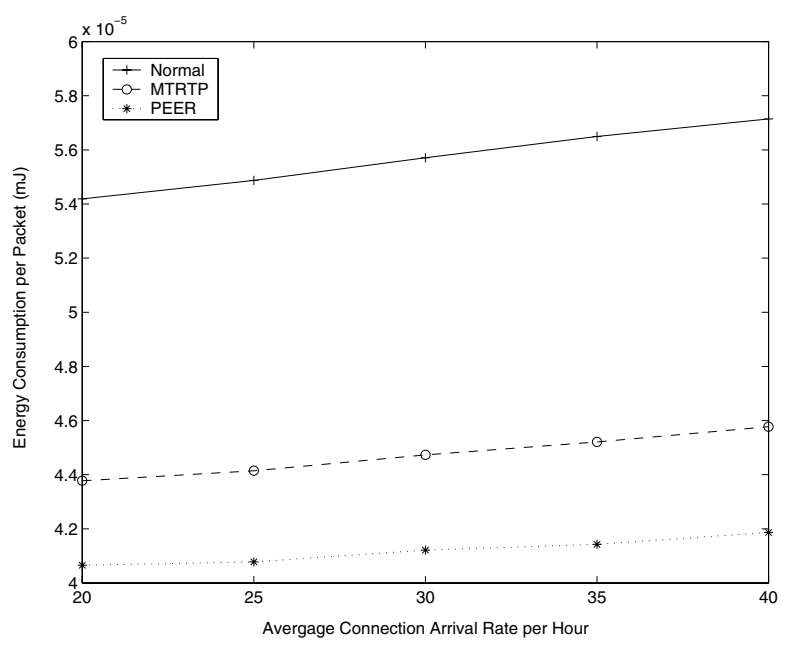

Fig. 12. Different Connection Arrival Rate (static).

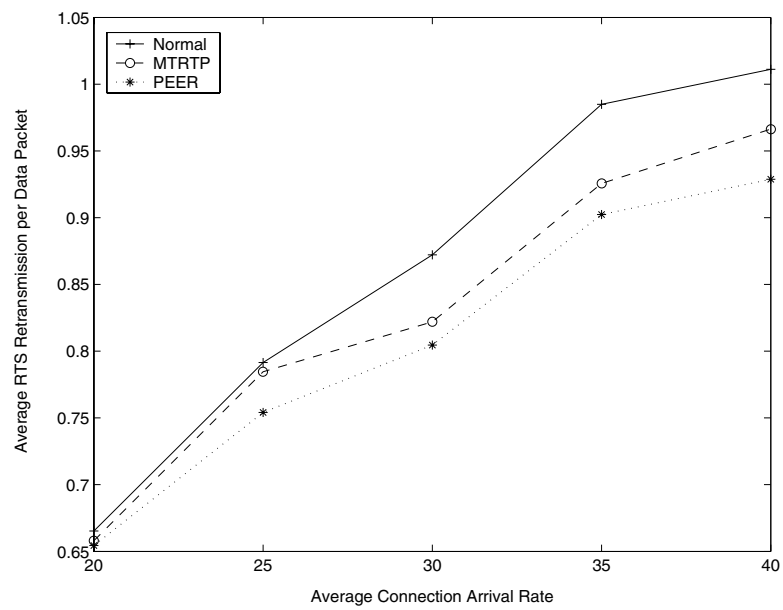

Fig. 13. Different Connection Arrival Rate (static).

destination nodes, and the total number of RTS retransmission for each simulations. The two metrics we used to evaluate the protocols are:

- Energy Consumption per Packet: It is defined by the total energy consumption divided by the total number of packets received. This metric reflects the energy efficiency for each protocol.

- Average RTS Retransmission per Data Packet: It is defined by the total number of RTS retransmission divided by the total number of packets received. As the RTS packet is transmitted at the maximum power level and the packet size is very small, most of RTS retransmission is because of collision. Therefore, this metric can reflect the collision rate for each protocol. Higher collision rate will cause more energy consumption, higher end-to-end delay, and lower throughput.

The simulation results are in Fig. 8-13. For all three different groups of studies, PEER protocol performs the best in terms of Energy Consumption per Packet as well as Average RTS Retransmission per Data Packet, followed by MTRTP protocol and normal protocol. 
Both PEER and MTRTP protocol search for energy efficient path instead of shortest path in normal protocol so that they can perform better in terms of energy consumption. PEER performs better than MTRTP in terms of energy consumption. There are several reasons for that. First, PEER protocol uses a more accurate link cost. Second, there are a lot of routing overhead in MTRTP that the route request packet from the most energy efficient path has higher probability of being lost in some intermediate node. Third, PEER protocol can adapt the path with the environment change quickly.

With power control scheme in all three protocols, RTS retransmission is mainly caused by asymmetric power. For normal protocol, the distance on each link can be quite different, ranging from very small up to the transmission range. While the two energy efficient routing protocols try to use some short distance links. Therefore, the retransmission rate is higher for normal protocol than the energy efficient routing protocols. As the link cost for MTRTP underestimates the real energy consumption, it tends to use larger number of hops. This will also increase the chance of RTS packets being lost and hence the retransmissions. So PEER protocol performs the best in terms of RTS retransmission rate.

It is interesting to observe that the RTS retransmission rate increases with the density in Fig. 9 for all protocols, while the energy consumption per packet in Fig. 8 has no such trend. This is because even though higher retransmission rate can cause more energy consumption, it can be compensated by the more energy efficient paths found by the routing protocols with higher number of nodes.

\section{Mobile Scenario}

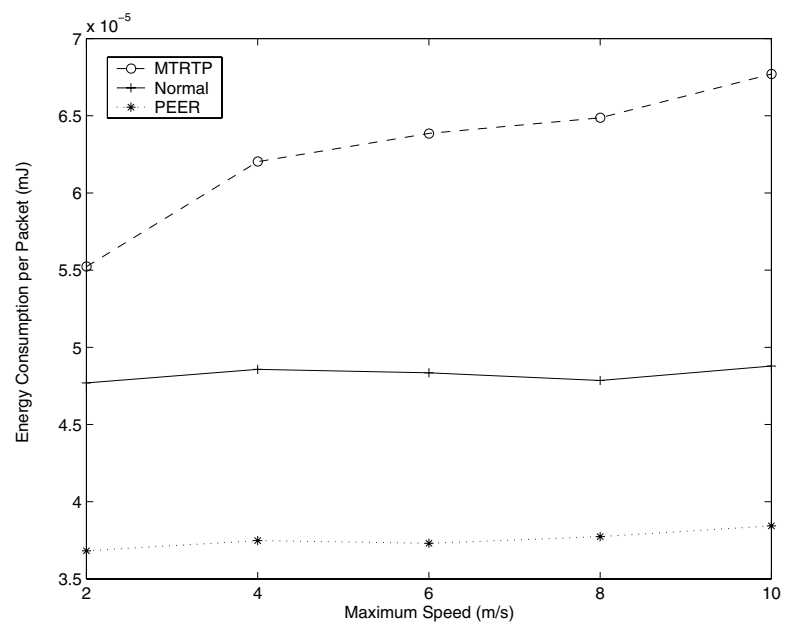

Fig. 14. Different speed (mobile).

For mobile scenario, we also studied the same metrics as in static scenarios for each protocol. And the three groups of simulations are different speed, different packet size, and different connection rate. The simulation results are in Fig. 1419. For all three different groups of studies, PEER protocol performs the best in terms of Energy Consumption per Packet as well as Average RTS Retransmission per Data Packet.

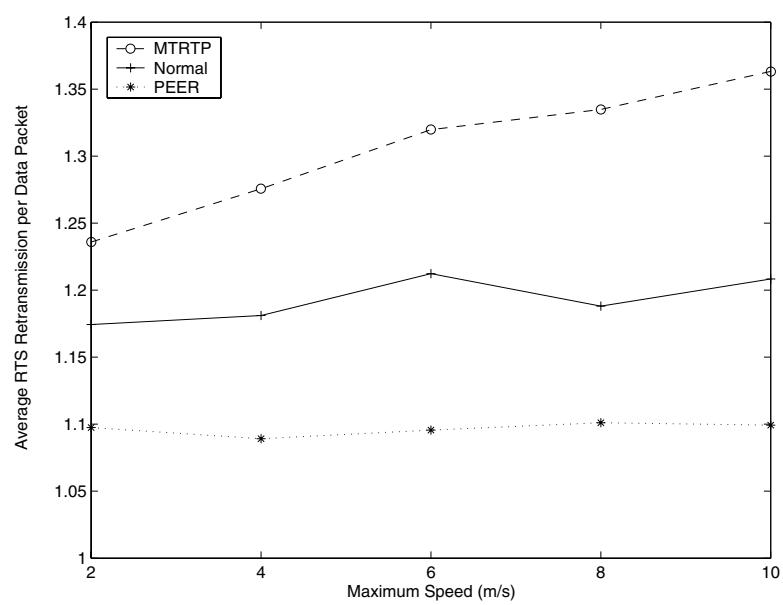

Fig. 15. Different speed (mobile).

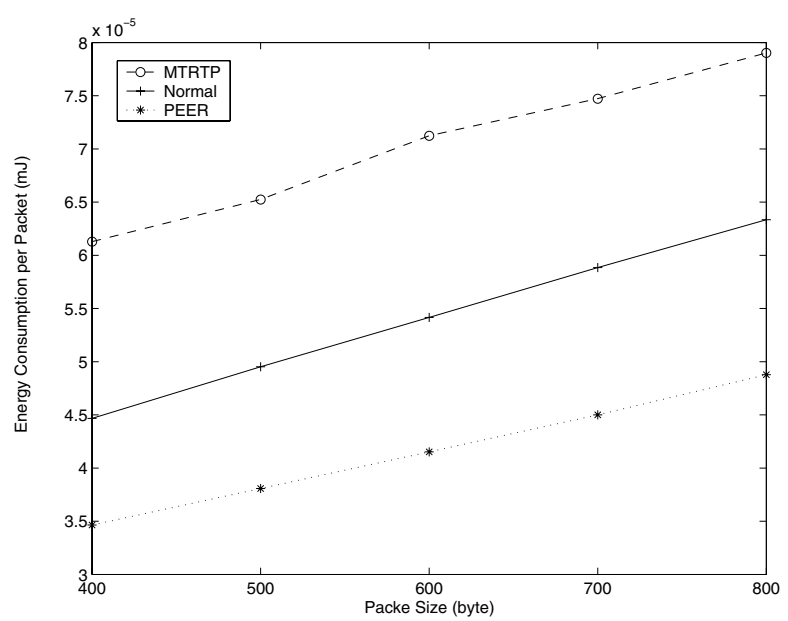

Fig. 16. Different packet size (mobile).

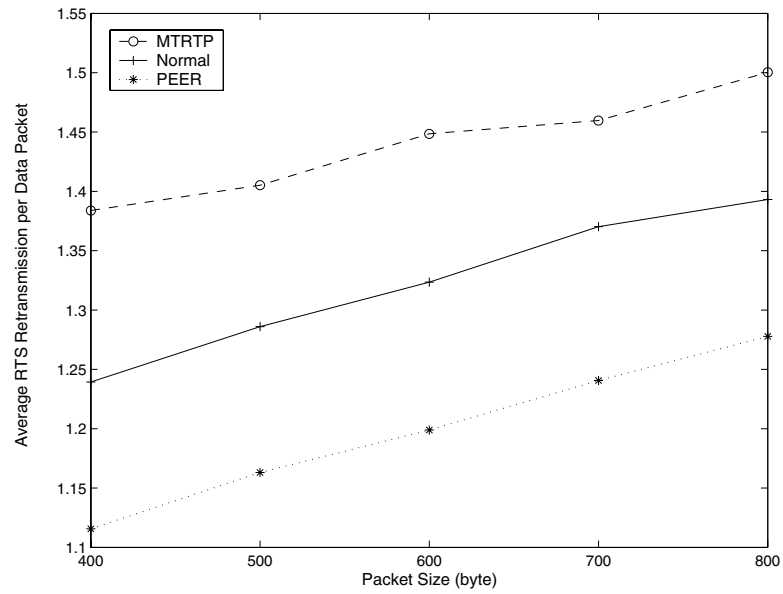

Fig. 17. Different packet size (mobile).

MTRTP performs the worst in terms of energy consumption, as its route maintenance scheme could not adapt with the mobility well. So the original minimum energy path would not be 


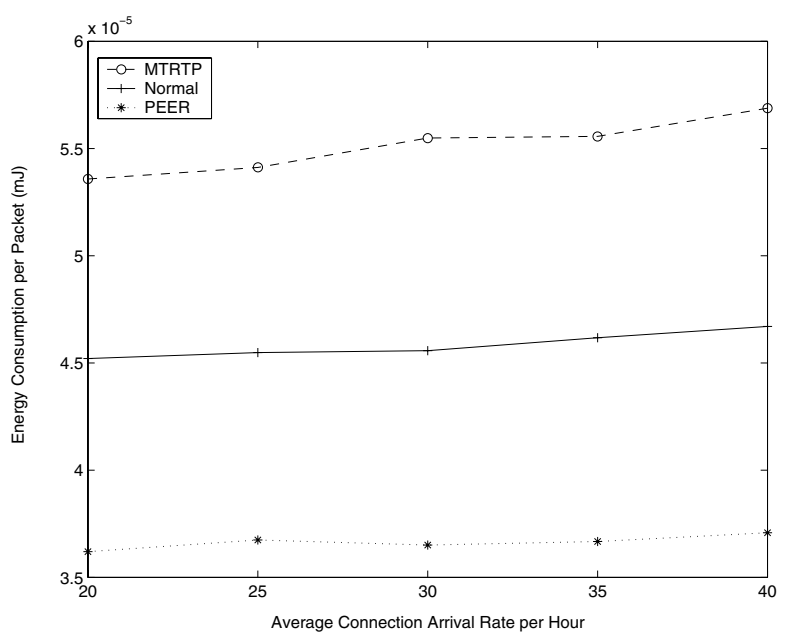

Fig. 18. Different Connection Arrival Rate (mobile).

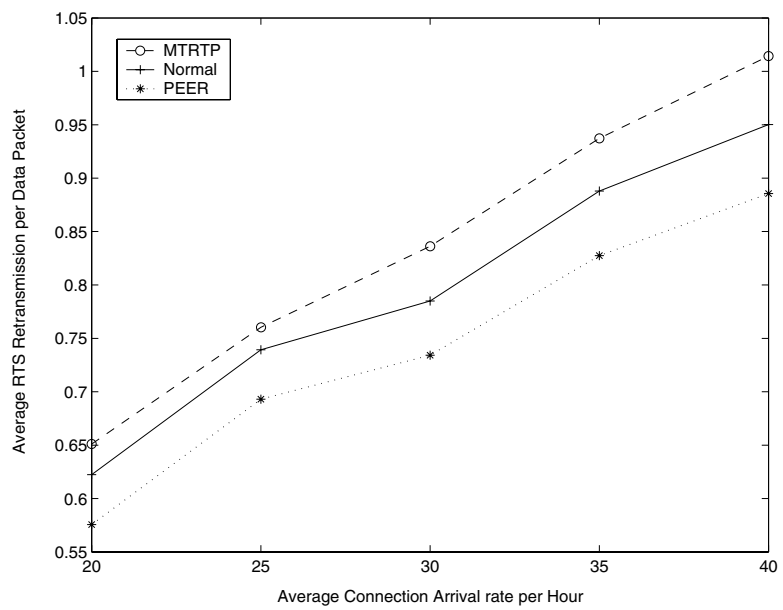

Fig. 19. Different Connection Arrival Rate (mobile).

energy efficient any more because of node mobility. MTRTP even consumes much more energy than normal protocol as its path normally has more hops. As PEER adapts the path with the mobility, it could get an energy efficient path all the time. Therefore, it performs much better than normal protocol and consumes several times lower energy as compared to MTRTP.

As mentioned in static scenarios, the RTS retransmission is mainly caused by asymmetric power. Because of node mobility, MTRTP will have similar asymmetric power issue as normal protocol now. In addition, due to larger number of hops, the RTS retransmission rate is larger for MTRTP than normal protocol. Again, because PEER protocol could adapt the path with the mobility, it still tries to use some short distance link in spite of node mobility. So it performs better than normal protocol.

\section{CONCLUSION}

In this paper, we first studied the high routing overhead, long setup time and maintenance issues associated with the minimum energy routing protocols. Based on these observations, we propose a progressive energy efficient routing (PEER) protocol, which achieves low routing overhead, short setup time, and is highly adaptive to the environment change. The simulation-based performance studies in static scenario as well as mobile scenario verify that our protocol performs better than other protocols.

\section{REFERENCES}

[1] K. Scott and N. Bambos, "Routing and Channel Assignment for Low Power Transmission in PCS", ICUPC '96, Oct. 1996

[2] S. Doshi, S. Bhandare, and T. X Brown, "An On-demand Minimum Energy Routing Protocol for a Wireless Ad Hoc Network", ACM Mobile Computing and Communications Review, vol. 6 , no. 3 , July 2002

[3] V. Rodoplu and T. Meng, "Minimum Energy Mobile Wireless Networks", IEEE Journal on Selected Areas on Communications, vol. 17, Aug. 1999.

[4] S. Banerjee and A. Misra, "Minimum Energy Paths for Reliable Communication in Multi-hop Wireless Networks", $M O B I$ HOC'02, June. 2002

[5] J. Gomez, A. T. Campbell, M. Naghshineh, and C. Bisdikian, "Conserving Transmission Power in Wireless Ad Hoc Networks", IEEE Conference on Network Protocols, Nov. 2001

[6] J. Zhu, C. Qiao and X. Wang, "A Comprehensive Minimum Energy Routing Protocol for Wireless Ad Hoc Networks", INFOCOM'04 , Mar. 2004

[7] C. K. Toh, H. Cobb and D. Scott, "Performance Evaluation of Battery-Life-Aware Routing Schemes for Wireless Ad Hoc Networks", ICC'01, June 2001

[8] A. Misra and S. Banerjee, "MRPC: Maximizing Network Lifetime for Reliable Routing in Wireless Environments", WCNC'02, Mar. 2002

[9] ANSI/IEEE Std 802.11, 1999 Edition

[10] E. Jung and N. H. Vaidya, "A Power Control MAC Protocol for Ad Hoc Networks", MOBICOM'02, Sept. 2002.

[11] G. Bianchi and I. Tinnirello, "Kalman Filter Estimation of the Number of Competing Terminals in an IEEE 802.11 network", INFOCOM'03, 2003

[12] C-K Toh, "Ad Hoc Mobile Wireless Networks Protocols and Systems", Prentice Hall, 2002

[13] T. H. Cormen, C. E. Leiserson and R. L. Rivest, "Introduction to Algorithms", MIT Press, 1998 